\title{
Left atrial localized low-voltage areas indicate whole left atrial electrophysiological degeneration in atrial fibrillation patients
}

\author{
Naoya Kurata $^{1}$, Masaharu Masuda ${ }^{1}$, Takashi Kanda ${ }^{1}$, Mitsutoshi Asai ${ }^{1}$, Osamu Iida ${ }^{1}$, Shin \\ Okamoto $^{1}$, Takayuki Ishihara ${ }^{1}$, Kiyonori Nanto ${ }^{1}$, Takuya Tsujimura ${ }^{1}$, Yasuhiro Matsuda ${ }^{1}$, \\ Yosuke Hata ${ }^{1}$, Hiroyuki Uematsu ${ }^{1}$, and Toshiaki Mano ${ }^{1}$ \\ ${ }^{1}$ Kansai Rosai Hospital
}

April 3, 2021

\begin{abstract}
Background: The efficacy of ablation targeting low-voltage areas (LVAs) is controversial, although LVA presence is well known to be associated with AF recurrence after ablation. Atrial fibrillation (AF) substrate may not localize within LVAs. Methods and results: This observational study enrolled 405 consecutive patients who underwent an initial AF ablation procedure. The left atrial voltage map was obtained after pulmonary vein isolation. LVAs were defined as areas with voltage $<0.5 \mathrm{mV}$. To estimate whole atrial electrophysiological degeneration, mean regional voltage at each of 6 regions and left atrial total conduction velocity were measured. LVAs existed in 143 of 405 (35.3\%) patients. Patients with LVAs demonstrated lower mean regional voltages throughout all 6 regions than those without LVAs $(1.3[1.8,0.8]$ vs. $0.6[1.0,0.2]$ for anterior wall, $\mathrm{p}<0.001)$. On the other hand, left atrial conduction velocity was lower in patients with LVAs than in those without (0.89 [1.01, 0.74] vs. 0.93 $[1.03,0.87] \mathrm{m} / \mathrm{s}, \mathrm{p}<0.001)$. Multivariate analysis revealed that low left atrial total conduction velocity and a higher number of regions with mean voltage reduction were independently associated with AF recurrence, although LVA presence was not. Conclusion: Patients with localized left atrial LVAs were characterized by whole left atrial electrophysiological degeneration as assessed by mean regional voltage and conduction velocity. In addition, whole left atrial electrophysiological degeneration parameters were well associated with AF recurrence.
\end{abstract}

Left atrial localized low-voltage areas indicate whole left atrial electrophysiological degeneration in atrial fibrillation patients.

Short title: localized LVA and whole atrial electrophysiological degeneration

Naoya Kurata1) BHS; Masaharu Masuda ${ }^{2)}$, MD, PhD; Takashi Kanda ${ }^{2}$, MD; Mitsutoshi Asai ${ }^{2}$, MD, $\mathrm{PhD}$; Osamu Iida ${ }^{2)}$, MD; Shin Okamoto ${ }^{2)}$, MD; Takayuki Ishihara ${ }^{2)}, \mathrm{MD}$; Kiyonori $\mathrm{Nanto}^{2)}$, MD; Takuya Tsujimura $^{2)}$, MD; Yasuhiro Matsuda ${ }^{2)}$, MD; Yosuke Hata ${ }^{2)}$, MD; Hiroyuki Uematsu' ${ }^{2)}$, MD; Toshiaki Mano ${ }^{2)}$, $\mathrm{MD}, \mathrm{PhD}$

\section{Authors' affiliations:}

Kansai Rosai Hospital Clinical engineer, 3-1-69 Inabaso, Amagasaki, Hyogo, Japan

Kansai Rosai Hospital Cardiovascular Center, 3-1-69 Inabaso, Amagasaki, Hyogo, Japan

Funding and conflicts of interest: Not is all authors.

Total word count: 3939

Figure and table : 4 figures and 3 tables

Supplemental figure: 2 figures 


\section{Corresponding author:}

Masaharu Masuda

Kansai Rosai Hospital Cardiovascular Center

3-1-69 Inabaso, Amagasaki, Hyogo 660-8511, Japan

Tel: $+81-6-6416-1221$

Fax: +81-6-6419-1870

E-mail:masuda-masaharu@kansaih.johas.go.jp

\section{Abstract}

Background: The efficacy of ablation targeting low-voltage areas (LVAs) is controversial, although LVA presence is well known to be associated with AF recurrence after ablation. Atrial fibrillation (AF) substrate may not localize within LVAs.

Methods and results: This observational study enrolled 405 consecutive patients who underwent an initial $\mathrm{AF}$ ablation procedure. The left atrial voltage map was obtained after pulmonary vein isolation. LVAs were defined as areas with voltage $<0.5 \mathrm{mV}$. To estimate whole atrial electrophysiological degeneration, mean regional voltage at each of 6 regions and left atrial total conduction velocity were measured. LVAs existed in 143 of $405(35.3 \%)$ patients. Patients with LVAs demonstrated lower mean regional voltages throughout all 6 regions than those without LVAs $(1.3[1.8,0.8]$ vs. $0.6[1.0,0.2]$ for anterior wall, $\mathrm{p}<0.001)$. On the other hand, left atrial conduction velocity was lower in patients with LVAs than in those without (0.89 [1.01, $0.74]$ vs. $0.93[1.03,0.87] \mathrm{m} / \mathrm{s}, \mathrm{p}<0.001)$. Multivariate analysis revealed that low left atrial total conduction velocity and a higher number of regions with mean voltage reduction were independently associated with AF recurrence, although LVA presence was not.

Conclusion: Patients with localized left atrial LVAs were characterized by whole left atrial electrophysiological degeneration as assessed by mean regional voltage and conduction velocity. In addition, whole left atrial electrophysiological degeneration parameters were well associated with AF recurrence.

Key words: atrial fibrillation; low-voltage areas; recurrence; ablation; conduction velocity; regional voltage

\section{Abbreviation}

$\mathrm{AF}=$ atrial fibrillation; PVI $=$ pulmonary vein isolation; LVA $=$ low-voltage areas; Receiver Operating Characteristic $=\mathrm{ROC}$

\section{Introduction}

Catheter ablation has become a mainstream treatment option for atrial fibrillation (AF). Electrical pulmonary vein isolation (PVI) is well established as the cornerstone of $\mathrm{AF}$ ablation. ${ }^{1,2}$ However, frequent $\mathrm{AF}$ recurrence after ablation remains an unsolved problem, with reported 1-year AF recurrence rates of 20$50 \% .^{2-4}$

The presence of left atrial low-voltage areas (LVAs) has been reported to be strongly associated with AF recurrence after ablation. ${ }^{5-7}$ Nevertheless, several randomized control trials evaluating the efficacy of LVA ablation in addition to PVI reported controversial results ${ }^{8-11}$ which suggested that extra-pulmonary-vein AF substrate does not always localized within LVAs.

The purpose of this study was to compare whole left atrial electrophysiological degeneration between patients with and without localize LVAs, and to explore clinical and mapping data associated with AF recurrence after ablation.

\section{Methods}

3.1 Patients 
This observational study enrolled 405 consecutive patients who underwent the initial AF ablation procedure at Kansai Rosai Hospital between Aug 2017 to Aug 2019. Patients in whom a voltage map was not obtained due to unstable cardiac rhythm and those whose $3 \mathrm{D}$ mapping was not the CARTO (CARTO ${ }^{\circledR}$; Biosense Webster, Diamond Bar, CA, USA) system were excluded. This study complied with the Declaration of Helsinki. Written informed consent for the ablation and for participation in the study was obtained from all patients, and the protocol was approved by our institutional review board.

\subsection{Ablation procedure}

Electrophysiological studies and catheter ablation were performed by three experienced operators (M.M, T.K, and Y.M) with the patient under intravenous sedation with dexmedetomidine. The operator performed the mapping and ablation under guidance with an electroanatomical mapping system (CARTO $3^{\circledR}$ ). First, PVI was performed using an open-irrigated ablation catheter (Thermocool SmartTouch ${ }^{\circledR}$, Biosense Webster), a cryoballoon (Arctic Front Advance ${ }^{\circledR}$, Medtronic, Minneapolis, MN, USA), or a laser balloon (HeartLight ${ }^{\circledR}$, CardioFocus, Marlborough, MA, USA). Radiofrequency application was set at $30 \mathrm{~W}$ using a dragging technique with a maximum temperature of $42{ }^{\circ} \mathrm{C}$ and an irrigation rate of $17 \mathrm{ml} / \mathrm{min}$. The operator attempted to maintain an appropriate contact force between the catheter and endocardium of 5 to $20 \mathrm{~g}$. In cases using a cryoballoon, 180-sec freezing was applied. The laser balloon was inflated with the goal of complete occlusion of the PV ostium. Laser lesions were created with a $30-50 \%$ lesion overlap. Where very good tissue contact was obtained, maximal power ( $12 \mathrm{~W}$ for 20 seconds) was chosen. At regions with moving blood, laser energy was applied at $7 \mathrm{~W}$ for 30 seconds. PV electrograms were recorded using a 20-pole circular mapping catheter (LassoNaV ${ }^{\circledR}$, Biosense Webstar). PVI was considered complete when both entrance and exit blocks were created.

\subsection{Left atrial voltage mapping}

Following PVI, detailed voltage mapping using a 20-pole circular catheter with 1-mm electrodes (LassoNaV ${ }^{\circledR}$, Biosense Webstar) or a 20-pole multielectrode catheter arranged in 5 soft radiating spines (Pentaray ${ }^{\circledR}$, Biosense Webster) was performed during 100-beat-per-minute paced rhythm from the high right atrium. Mapping points were automatically acquired using the CARTO confidence module with the following settings: cycle length filtering, $\pm 30 \mathrm{msec}$; localize activation time stability, $<3 \mathrm{msec}$; position stability, $<2 \mathrm{~mm}$; and density, $<1 \mathrm{~mm}$. Left atrial geometry was created using the fast-anatomical mapping module. Mapping was continued to fill all color gaps on the voltage map with an interpolation threshold of $17 \mathrm{~mm}$ for fill threshold and $10 \mathrm{~mm}$ for color threshold. If poor contact between the circular mapping catheter and endocardium surface was suspected, mapping using the ablation catheter was added with a point acquisition setting of contact force [?] $5 \mathrm{~g}$. The band pass filter was set at 30 to $500 \mathrm{~Hz}$.

LVAs were defined as areas with a bipolar peak-to-peak voltage $<0.50 \mathrm{mV}$ covering $>5 \mathrm{~cm}^{2}$ of left atrium. On the voltage map, the bipolar voltage color bar was set to range from 0.10 to $0.50 \mathrm{mV}$ and scar level was set at $<0.05 \mathrm{mV}$. The left atrium was divided into six regions - septal, anterior, roof, posterior, inferior, and lateral - as reported previously. ${ }^{5}$

After this procedure, constant burst pacing was performed for $5 \mathrm{~s}$ at each cycle length, starting with 300 $\mathrm{ms}$ and a subsequent decrement of 20 to $200 \mathrm{~ms}$ or the shortest cycle length that resulted in 1:1 atrial capture. This was followed by a high-dose isoproterenol provocation test (infusion of 5,10 , and $20 \mu \mathrm{g} / \mathrm{min}$ isoproterenol for $2 \mathrm{~min}$ each) to induce $\mathrm{AF}$ or atrial tachycardia. If atrial flutters or non-PV $\mathrm{AF}$ triggers were observed spontaneously or induced by atrial burst stimuli or isoproterenol infusion, additional ablation were performed. Ablation of induced and spontaneously developing AF-triggering ectopies and atrial tachycardia was attempted at the earliest activation site for AF trigger or centrifugal atrial tachycardia, and across the reentrant circuit for macro-reentrant atrial tachycardia. Ablation targeting LVAs, linear ablations and/or ablation guided by complex fractionated electrograms were performed at the discretion of attending operators.

\subsection{Whole left atrial electrophysiological degeneration}


Whole left atrial electrophysiological degeneration was assessed by the mean regional voltage at each region and left atrial total conduction velocity. ${ }^{12}$

Mean regional voltage was calculated by averaging 10 points evenly selected across the region. ROC analysis was used to estimate a best cut-off value of mean regional voltage to predict AF recurrence. The extension of mean regional voltage reduction was assessed by the number of regions with a mean regional voltage $<$ the region-specific cut-off value.

Left atrial conduction velocity was calculated as left atrial anterior conduction distance divided by conduction time between the start (septum) and end of the propagation wave front (lateral mitral annulus) in the left atrium, as reported previously. ${ }^{12}$ Anterior conduction distance was measured manually by tracing the pathway of the propagation wave front from the start point to the end point in the anterior left atrium.

\subsection{Post-procedure follow-up}

Patients were discharged two days after ablation if their clinical status was stable. Following a blanking period of 3 months, they completed outpatient clinical visits and 12-lead ECG monitoring at 1, 3,6 and 12 months, and 24 h-Holter ECG monitoring every 6 months. Additional Holter monitoring was performed if arrhythmic symptoms occurred. Either of the following events following the initial 3 months after ablation (blanking period) was considered to indicate AF recurrence: (1) atrial tachyarrhythmias recorded on routine or symptom-triggered ECG during an outpatient visit; or (2) atrial tachyarrhythmias of at least a 30 seconds' duration on ambulatory ECG monitoring. All antiarrhythmic drugs were discontinued 3 months after the procedure, unless AF recurrence was diagnosed.

\subsection{Statistical analysis}

Categorical values are expressed as absolute values and relative frequencies. Continuous variables are expressed as mean $\pm \mathrm{SD}$. Tests for significance were conducted using the unpaired t-test or nonparametric test (Mann-Whitney U-test) for continuous variables and the chi-squared test or Fisher's exact test for categorical variables. The cutoff value of the voltage in each region was examined using the Receiver Operating Characteristic (ROC) curve. The score below the cutoff value was set as 1 point each, and the relationship with the recurrence of atrial fibrillation was examined by a chi-square test. Clinical factors associated with AF recurrence were determined using univariate and multivariate Cox proportional hazard models and logistic regression analyses. All statistical analyses were performed using commercial software (SPSS version 25 (), SPSS, Chicago, IL, USA), and statistical significance was defined as $\mathrm{p}<0.05$.

\section{Results}

\subsection{Baseline and procedure characteristics}

Patient characteristics are shown in Table 1. LVAs existed in 143 of 405 (35.3\%) patients. Patients with LVAs were older, more likely to be female, had a higher $\mathrm{CHA}_{2} \mathrm{DS}_{2}$-VASc score, and larger left atrial diameter. Heart failure, pacemaker implantation, and non-paroxysmal AF were more common in patients with LVAs than in those without.

Ablation lesions created during the initial ablation are shown in Table 2. PVI was completed in all patients. There was no difference in modalities used for PVI between patients with and without LVAs (radiofrequency catheter, $68 \%$ vs. $78 \%$; cryoballoon catheter, $31 \%$ vs. $20 \%$; laser balloon catheter, $1 \%$ vs. $1 \%$; p=0.06). No severe procedure-related complications such as cardiac tamponade, stroke, esophageal injury, or major bleeding were observed. Patients with LVAs more frequently underwent additional ablations such as LVA ablation and left atrial linear ablations.

\subsection{LVA presence and whole left atrial electrophysiological degeneration}

Total LVA size among the 143 patients with LVAs was $12.8 \pm 8.6 \mathrm{~cm}^{2}$. The distribution of LVAs was the anterior region in $75 \%$; septal region in $69 \%$; roof region in $41 \%$; posterior region in $32 \%$; bottom region in $46 \%$, and lateral region in $7 \%$. 
Whole left atrial electrophysiological degeneration was assessed by mean regional voltages and left atrial total conduction velocity. Patients with LVAs demonstrated lower mean regional voltages throughout all 6 regions than those without LVAs (Figure 1). Example cases showing the absence or presence of LVAs $(<0.5$ $\mathrm{mV}$ ) and whole left atrial voltage reduction are presented in Supplementary Figure 1. In contrast, a longer left atrial total conduction time and lower left atrial total conduction velocity were demonstrated in patients with LVAs than in those without (Figure 2).

\subsection{AF recurrence using left atrial regional voltage value}

During a mean follow-up period of $16 \pm 8$ months, 126 (31\%) patients experienced AF recurrence. Patients with AF recurrence had LVAs more frequently than those without ( $53 \%$ vs. 27\%, p<0.001).

Associations between AF recurrence and mapping parameters representing whole left atrial electrophysiological degeneration were explored. Mean regional voltages were significantly lower in patients with AF recurrence than in those without in all 6 regions (Figure 3). Cut-off values of regional voltage obtained by $\mathrm{ROC}$ analysis for the prediction of AF recurrence were $0.9 \mathrm{mV}$ for the anterior and septal regions, $1.0 \mathrm{mV}$ for the roof region, $1.2 \mathrm{mV}$ for the posterior region, $1.4 \mathrm{mV}$ for the bottom region, and $1.8 \mathrm{mV}$ for the lateral region) (Supplementary Figure 2). Regions with a mean voltage $<$ the region-specific cut-off value were defined as a mean regional voltage reduction. AF recurrence rates stratified according to the number of regions with mean voltage reduction are presented in Figure 4. AF recurrence rates became higher as the number of regions with a mean voltage reduction increased. On the other hand, left atrial total conduction velocity was significantly lower in patients with $\mathrm{AF}$ recurrence than in those without $(0.84 \pm 0.15$ vs. 0.98 $\pm 0.17 \mathrm{~m} / \mathrm{s}, \mathrm{p}<0.005)$.

Predictors of AF recurrence are presented in Table 3. On the univariate analysis, patients with AF recurrence more frequently had non-paroxysmal AF and left atrial LVAs, and demonstrated a higher left atrial diameter, lower left ventricular ejection fraction, lower left atrial anterior conduction velocity, and higher number of regions with mean voltage reduction. Multivariate analysis revealed that low left ventricular ejection fraction, low left atrial conduction velocity, and a high number of regions with mean voltage reduction were independently associated with AF recurrence.

\section{Discussion}

In this observational study, we evaluated 405 patients who underwent initial AF ablation, and compared whole left atrial electrophysiological degeneration assessed by electroanatomical mapping parameters between patients with and without LVAs. We then explored clinical and mapping parameters predicting AF recurrence. The main findings were (1) patients with left atrial LVAs had a lower mean regional voltage throughout all 6 regions, and regional mean voltage reduction was more extensive than those without LVAs; (2) left atrial total conduction velocity was lower in patients with LVAs than in those without; and (3) mapping parameters representing whole left atrial electrophysiological degeneration such as left atrial total conduction velocity and the extension of mean regional voltage reduction independently predicted AF recurrence. To our knowledge, this is the first clinical study to explore the association between the presence of localized LVAs and whole left atrial electrophysiological degeneration.

\subsection{Association between localized LVA presence and whole left atrial electrophysiological degeneration.}

Patients with localized LVAs demonstrated whole left atrial degeneration, represented by an extensive mean regional voltage reduction and a low left atrial total conduction velocity. Several upstream factors causing atrial fibrosis have been reported, including atrial stretch, aging, AF burden, hypertension, heart failure, alcohol, obesity, and obstructive sleep apnea. ${ }^{13-17}$ These upstream factors would promote atrial fibrosis not only at localized areas, but also across the entire atrial myocardium. Therefore, when the voltages at some portions of the left atrium were below $0.50 \mathrm{mV}$ - which is a commonly used cut-off value of LVA - voltages at other areas likely decreased in parallel, even if not below $0.50 \mathrm{mV}$.

Myocardial degeneration of the entire atrium in $\mathrm{AF}$ patients has also been reported. Teh et al showed that AF patients had lower mean voltage, slower conduction, and more prevalent complex signals at a majority 
of left atrial regions than controls. ${ }^{13}$

\subsection{AF substrate extending to the whole left atrium}

In this and our previous study, ${ }^{12}$ whole left atrial electrophysiological abnormalities such as an extensive mean regional voltage reduction and a slow left atrial total conduction velocity were shown to be reliable predictors of $\mathrm{AF}$ recurrence.

In addition to the previously reported limited efficacy of LVA ablation, ${ }^{8,11}$ these results suggest the important clinical implication that AF substrate is not always limited to LVAs. Several substrate-based ablations using different ablation targets have been proposed. One study reported that the cut-off value of voltage for the prediction of fibrosis differs between regions, ${ }^{18}$ and others have shown that the use of different voltage criteria improves the efficacy of LVA ablation. ${ }^{19,20}$ The efficacy of ablation targeting fractionated electrograms during sinus rhythm using an ultra-high resolution mapping system has also been proposed. ${ }^{21}$

The management of AF based on therapies for upstream factors which promote atrial degeneration, such as hypertension, diabetes mellitus, and heart failure, will likely benefit by considering that the AF substrate extends across the whole atria rather than being localized to specific portions.

\subsection{Clinical implications}

The presence of a low-voltage area is a marker of myocardial damage, and in the left atrium where this findings is also locally observed, electrophysiological degeneration may already be present throughout the left atrium. Localized LVA suggests that it is a good maker of AF substrate, but may not always be an optimal ablation target.

\section{Study limitations}

Several limitations of the present study warrant mention. First, data analyses were not performed at independent facilities blinded to patient characteristics, which may have resulted in bias in mapping-data collection. In particular, mean regional voltage was obtained using arbitrarily selected mapping points within the region, possibly resulting in biased values. Second, the study did not investigate data from repeat procedures, and did not examine the reconnection rate of isolated pulmonary vein. Accordingly, AF recurrence does not necessarily mean that the patient had an extra-pulmonary-vein AF substrate. Third, ablation strategies were at the discretion of attending physician, possibly affecting rhythm outcomes. Fourth, AF recurrence after discharge was quantified on the basis of intermittent ECG monitoring, giving rise to the possibility that asymptomatic episodes of AF might have been missed. Finally, the study was conducted under a single-center, observational design. Confirmation awaits a prospective multicenter study.

\section{Conclusion}

Patients with localized left atrial LVAs had whole left atrial electrophysiological degeneration as assessed by regional mean regional voltages and total left atrial conduction velocity. In addition, whole left atrial electrophysiological degeneration parameters were well associated with AF recurrence. These findings suggest that localized LVA is an excellent maker of AF substrate, but may not always be an optimal ablation target.

\section{Author disclosure}

None.

\section{Acknowledgements}

We wish to express our sincerest appreciation to our staff colleagues for their continuing support and constant encouragement of these works.

\section{References}

1. Di Biase L, Elayi CS, Fahmy TS, et al. Atrial fibrillation ablation strategies for paroxysmal patients: randomized comparison between different techniques. Circ Arrhythm Electrophysiol 2009; 2: 113-119. 
2. Takahashi A, Iesaka Y, Takahashi Y, et al. Electrical connections between pulmonary veins: implication for ostial ablation of pulmonary veins in patients with paroxysmal atrial fibrillation. Circulation 2002; 105: 2998-3003.

3. Calkins H, Hindricks G, Cappato R, et al. 2017 HRS/EHRA/ECAS/APHRS/SOLAECE expert consensus statement on catheter and surgical ablation of atrial fibrillation. Heart Rhythm 2017; 14: e275-e444.

4. Verma Atul, Jiang Chen-yang, Betts Timothy R, et al. Approaches to catheter ablation for persistent atrial fibrillation. N Engl J Med 2015; 372: 1812-1822.

5. Rolf S, Kircher S, Arya A, Eitel C, Sommer P, Richter S, Gaspar T, Bollmann A, Altmann D, Piedra $\mathrm{C}$, et al. Tailored atrial substrate modification based on low-voltage areas in catheter ablation of atrial fibrillation. Circ Arrhythm Electrophysiol 2014; 7: 25-833.

6. Masuda M, Fujita M, Iida O, et al. Left atrial low-voltage areas predict atrial fibrillation recurrence after catheter ablation in patients with paroxysmal atrial fibrillation. Int J Cardiol 2018; 257: 97-101.

7. Vlachos K, Efremidis M, Letsas KP, et al. Low-voltage areas detected by high-density electroanatomical mapping predict recurrence after ablation for paroxysmal atrial fibrillation. J Cardiovasc Electrophysiol 2017; 28: 1393-1402.

8. Masuda M, Asai M, Iida O, et al. Additional Low-Voltage-Area Ablation in Patients With Paroxysmal Atrial Fibrillation: Results of the Randomized Controlled VOLCANO Trial. J Am Heart Assoc 2020; 9: $\mathrm{e} 015927$.

9. Kircher S, Arya A, Altmann D, et al. Individually tailored vs. standardized substrate modification during radiofrequency catheter ablation for atrial fibrillation: a randomized study. Europace 2018; 20: 1766-1775.

10. Yang B, Jiang C, Lin Y, et al. STABLE-SR (Electrophysiological Substrate Ablation in the Left Atrium During Sinus Rhythm) for the Treatment of Non paroxysmal Atrial Fibrillation: A Prospective, Multicenter Randomized Clinical Trial. Circ Arrhythm Electrophysiol. 2017; 10. pii: e005405. doi:10.1161/CIRCEP.117.005405.

11. Kumagai K, Toyama H, Zhang B. Effects of additional ablation of low-voltage areas after Box isolation for persistent atrial fibrillation. J Arrhythmia 2019; 35:737-738.

12. Kurata N, Masuda M, Kanda T, et al. Slow whole left atrial conduction velocity after pulmonary vein isolation predicts atrial fibrillation recurrence. J Cardiovasc Electrophysiol 2020; 31: 1942-1949.

13. Teh A, Kistler P, Lee G,et al. Electroanatomic remodeling of the left atrium in paroxysmal and persistent atrial fibrillation patients without structural heart disease. J Cardiovasc Electrophysiol 2012; 23: 232-238.

14. Kistler P, Sanders P, Fynn S, et al. Electrophysiologic and electroanatomic changes in the human atrium associated with age. J Am Coll Cardiol 2004; 44: 109-116.

15. Wong CX, Abed HS, Molaee P, et al. Pericardial fat is associated with atrial fibrillation severity and ablation outcome. J Am Coll Cardiol 2011; 57: 1745-1751.

16. Shahar E, Whitney CW, Redline S, et al. Sleep-disordered breathing and cardiovascular disease: crosssectional results of the Sleep Heart Health Study. Am J Respir Crit Care Med 2001; 163: 19-25.

17. Voskoboinik A, Prabhu S, Ling LH, Kalman JM, Kistler PM. Alcohol and Atrial Fibrillation: A Sobering Review. J Am Coll Cardiol 2016; 68: 2567-2576.

18. Kapa S, Desjardins B, Callans DJ, Marchlinski FE, Dixit S. Contact electroanatomic mapping derived voltage criteria for characterizing left atrial scar in patients undergoing ablation for atrial fibrillation. J Cardiovasc Electrophysiol 2014; 25:1044-1052.

19. Yagishita A, Gimbel JR, DE Oliveira S, et al. Long-Term Outcome of Left Atrial Voltage-Guided Substrate Ablation During Atrial Fibrillation: A Novel Adjunctive Ablation Strategy. J Cardiovasc Electrophysiol 2017; 28: 147-155.

20. Jadidi AS, Duncan E, Miyazaki S, et al. Functional nature of electrogram fractionation demonstrated by left atrial high-density mapping. Circ Arrhythm Electrophysiol 2012; 5: 32-42.

21. Hirokami J, Hiroshima K, Yamaji K, et al. Relationship between fractionated signal areas in the atrial muscle during sinus rhythm and atrial pacing and non-pulmonary vein foci: novel mapping strategy. Circ Arrhythm Electrophysiol 2020; 13: e008667. 
Figure legends

Figure 1. Comparison of mean regional voltage between patients with and without low-voltage areas

Mean regional voltages between patients with and without low-voltage areas were compared. Low-voltage areas indicated localized areas with bipolar voltage $<0.50 \mathrm{mV}$. Mean regional voltage was calculated by averaging evenly selected 10 points at the region. The left atrium was divided into 6 regions. Patients with localized low-voltage areas at any portion of the left atrium had significantly lower mean regional voltage throughout all regions than those without.

Figure 2. Comparison of left atrial whole conduction time and velocity between patients with and without low-voltage areas

Left atrial whole conduction time and velocity between patients with and without low-voltage areas were compared. Conduction time was defined as the time difference between the start (septum) and end of the left atrial propagation wave front (lateral mitral annulus) during right atrial pacing or sinus rhythm. Left atrial conduction velocity was calculated as left atrial anterior conduction distance divided by conduction time. Anterior conduction distance was measured manually by tracing the pathway of the propagation wave front from the start point to the end point in the anterior left atrium. Patients with localized low-voltage areas at any portion of the left atrium demonstrated longer conduction time and a slower conduction velocity than those without.

Figure 3. Comparison of mean regional voltage between patients with and without recurrence of atrial fibrillation

Mean regional voltages between patients with and without recurrence of atrial fibrillation were compared. Mean regional voltage was calculated by averaging 10 evenly selected points in the region. The left atrium was divided into 6 regions. Patients with recurrence of atrial fibrillation demonstrated lower mean regional voltages throughout all regions than those without.

Figure 4.Recurrence rates of atrial fibrillation stratified according to the number of regions with mean voltage reduction

Recurrence rates of atrial fibrillation stratified according to the number of regions with mean voltage reduction were compared. A region with mean voltage reduction was defined as a region with a mean voltage $<$ the region-specific cut-off value obtained using receiver-operator characteristics analysis for the prediction of atrial fibrillation recurrence $(0.9 \mathrm{mV}$ for anterior and septal, $1.0 \mathrm{mV}$ for roof, $1.2 \mathrm{mV}$ for posterior, $1.4 \mathrm{mV}$ for bottom, and $1.8 \mathrm{mV}$ for lateral). Recurrence rates became higher as the number of regions with mean voltage reduction increased.

Supplementary Figure 1. Representative cases showing the extension of voltage reduction with and without low-voltage areas

Left atrial voltage maps using various voltage thresholds with and without localized low-voltage areas are shown. Low-voltage areas indicated localized areas with bipolar voltage $<0.50 \mathrm{mV}$. A patient without lowvoltage areas (Upper) demonstrated no or smaller areas with reduced voltage even using a relatively high voltage threshold $(<1.0 \mathrm{mV}$, and $<1.5 \mathrm{mV})$ than a patient with localized low-voltage areas (lower).

Supplementary Figure 2. Receiver-operator characteristics curves of mean regional voltages for the prediction of recurrence of atrial fibrillation

The best cut-off value of mean regional voltage at each region for the prediction of recurrence of atrial fibrillation was calculated using the receiver-operator characteristics curve. AUC indicates area under the curve; CI, confidence interval. 



B. Left atrial conduction velocity
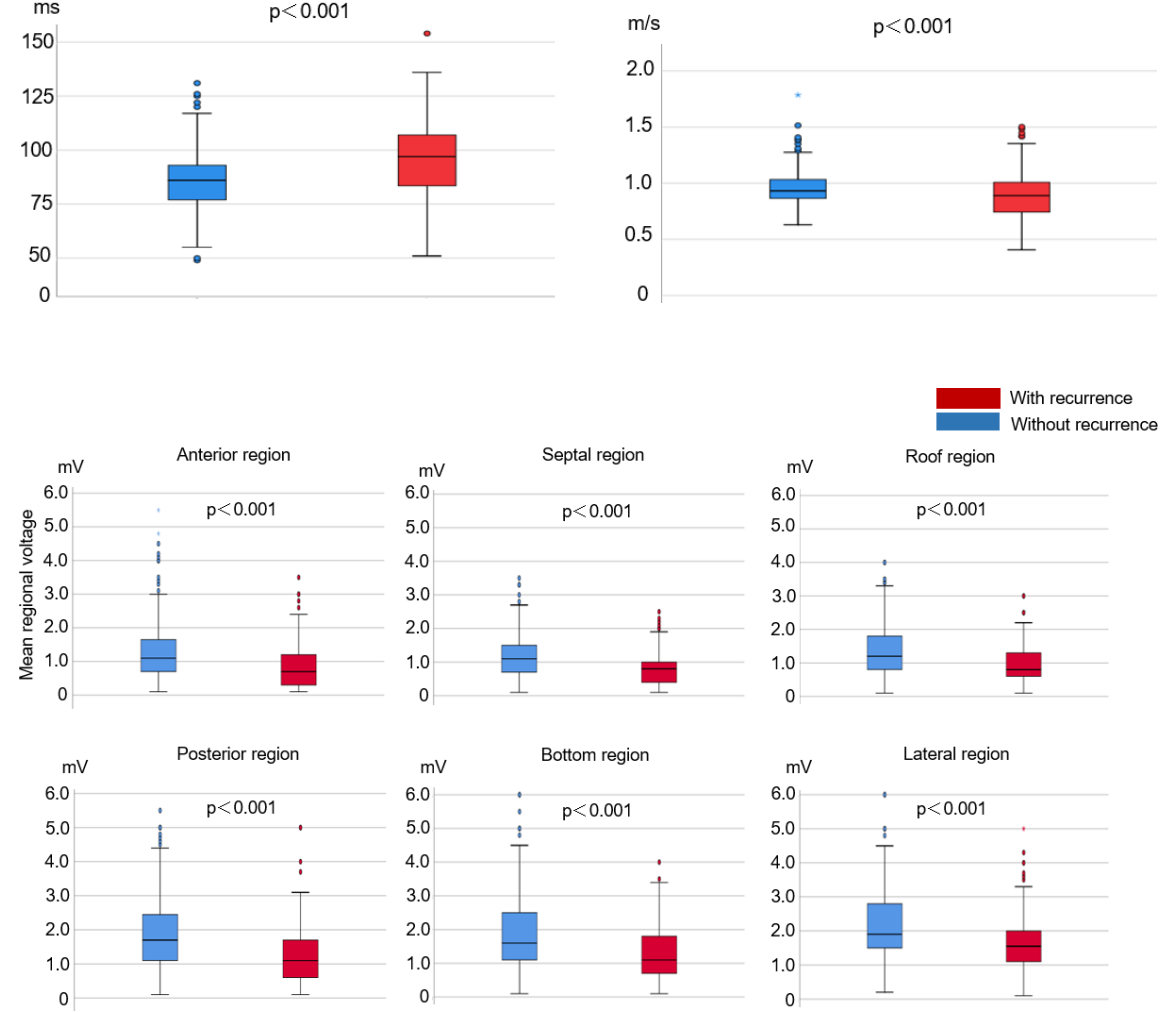


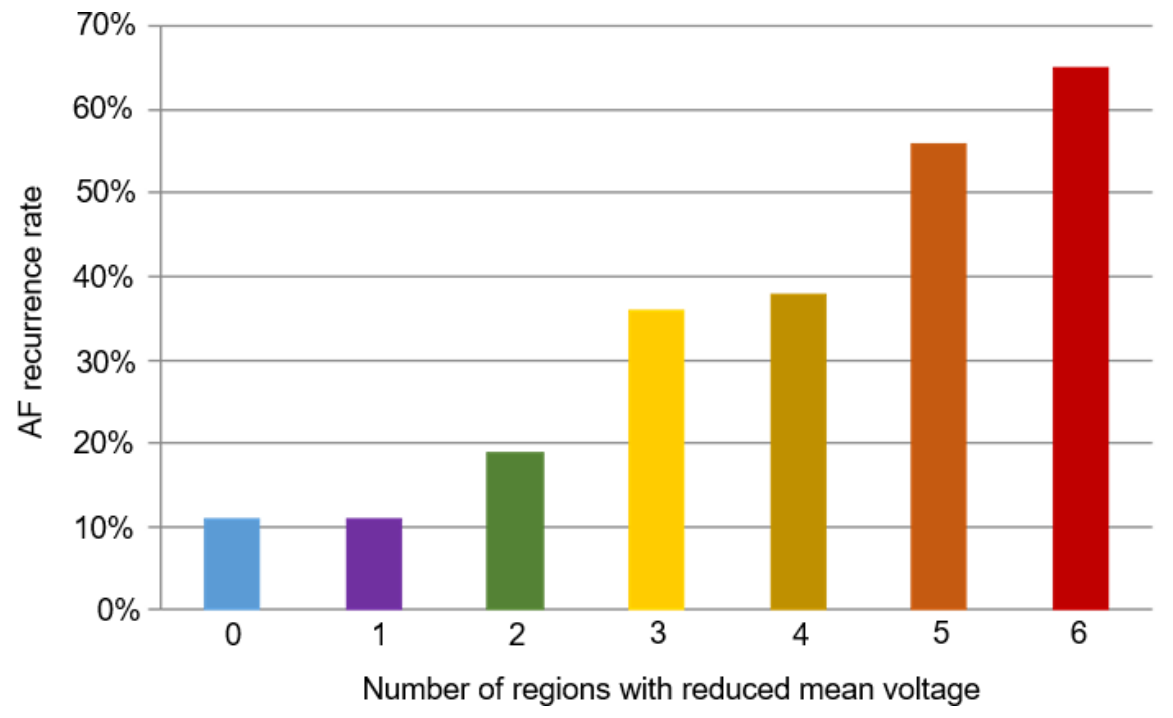

Hosted file

Table.pdf available at https://authorea.com/users/311161/articles/516569-leftatrial-localized-low-voltage-areas-indicate-whole-left-atrial-electrophysiologicaldegeneration-in-atrial-fibrillation-patients 